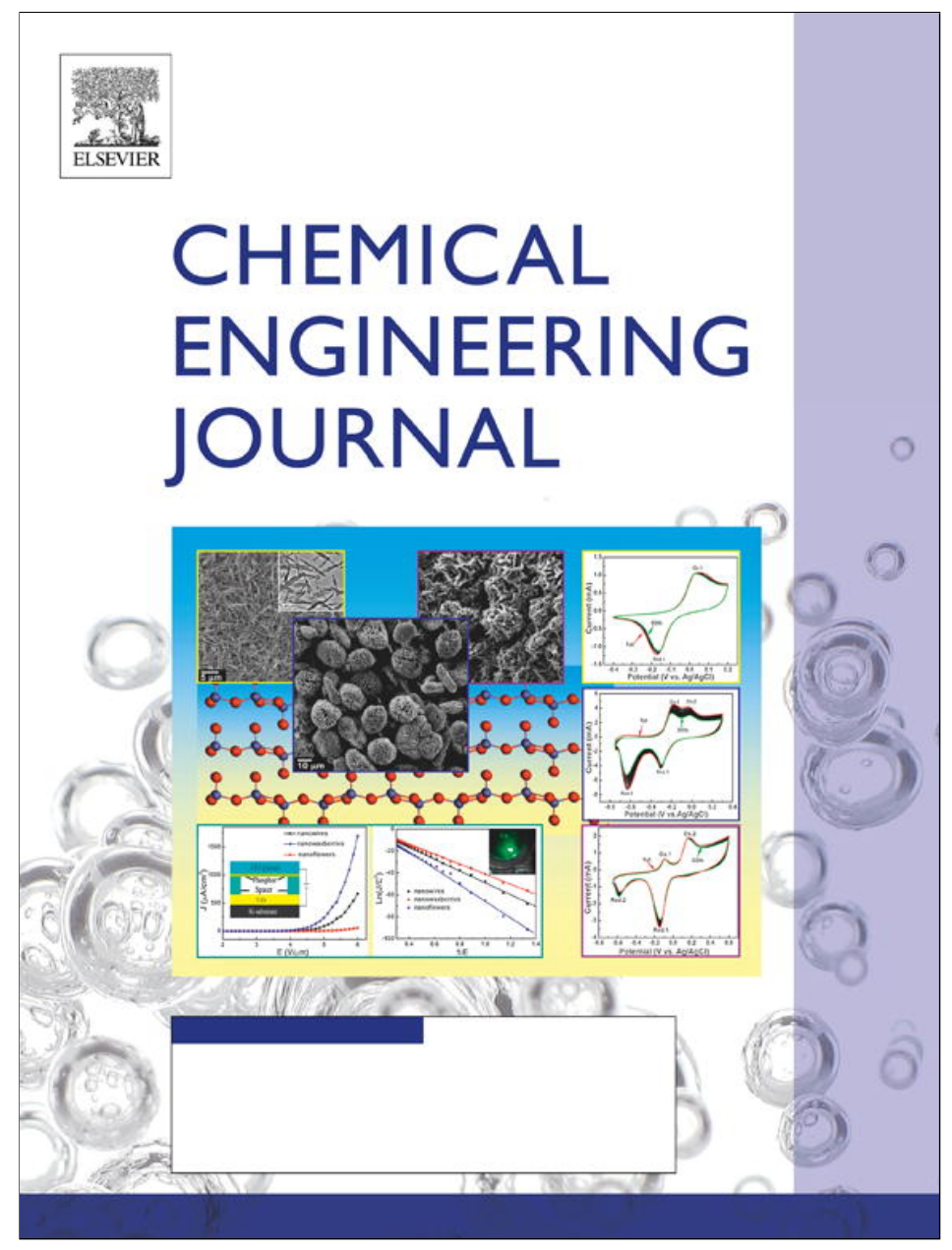

This article appeared in a journal published by Elsevier. The attached copy is furnished to the author for internal non-commercial research and education use, including for instruction at the authors institution and sharing with colleagues.

Other uses, including reproduction and distribution, or selling or licensing copies, or posting to personal, institutional or third party websites are prohibited.

In most cases authors are permitted to post their version of the article (e.g. in Word or Tex form) to their personal website or institutional repository. Authors requiring further information regarding Elsevier's archiving and manuscript policies are encouraged to visit: 


\title{
A novel vortex mixer actuated by one-shot electricity-free pumps
}

\author{
An-Shik Yang ${ }^{\mathrm{a}}$, Yi-Fan Hsieh ${ }^{\mathrm{b}}$, Long-Sheng Kuo ${ }^{\mathrm{b}}$, Li-Yu Tseng ${ }^{\mathrm{c}}$, Shao-Kai Liao ${ }^{\mathrm{b}}$, Ping-Hei Chen ${ }^{\mathrm{b}, *}$ \\ ${ }^{a}$ Department of Energy and Refrigerating Air-Conditioning Engineering, National Taipei University of Technology, Taipei 106, Taiwan, ROC \\ ${ }^{\mathrm{b}}$ Department of Mechanical Engineering, National Taiwan University, Taipei 106, Taiwan, ROC \\ ${ }^{c}$ Institute of Mechatronic Engineering, National Taipei University of Technology, Taipei 106, Taiwan, ROC
}

\section{H I G H L I G H T S}

- This study presents the design, fabrication and operation of a simple vortex mixer.

- The geometrically simple vortex mixer actuated by one-shot electricity-free pumps.

- A hemisphere-shaped vortex mixer merely needs $0.1 \mathrm{~s}$ to attain mixing index of $93 \%$.

- The mixer maintains a good mixing performance during the period of $0.1-0.4 \mathrm{~s}$.

\section{A R T I C L E I N F O}

\section{Article history:}

Received 9 February 2013

Received in revised form 3 May 2013

Accepted 14 May 2013

Available online 21 May 2013

\section{Keywords:}

Microfluidic

Computational fluid dynamics

Vortex mixer

\begin{abstract}
A B S T R A C T
Effective mixing is vitally important to many microfluidic devices in the areas of biotechnical industries, analytic chemistry and medical industries. However, most micro-mixers require complicated fabrication procedures, possibly inappropriate for practical microfluidic utilization. These mixers generally operate under low Reynolds-number conditions, causing a relatively long reaction time in various biochemical processes. This study presents a novel hemisphere-shaped vortex mixer to rapidly mix two liquids with simple geometric configuration. To simulate mixing behavior, computational analysis is based on the transient three-dimensional conservation equations of mass, momentum and species concentration. The test flow are treated as laminar, incompressible, miscible, uniform-property flows with insignificant gravity and temperature variation effects over the calculation domain. Considering the proposed mixer concurrently actuated by two finger-pressed pumps without electrical power, both experimental and simulation results show an intense swirling eddy generated in the core region of mixing chamber, achieving a mixing index up to $93 \%$ in a one-shot mixing event.
\end{abstract}

() 2013 Elsevier B.V. All rights reserved.

\section{Introduction}

Microfluidic devices have great potential in applications involving several micro-total analytic systems ( $\mu$-TAS). Among these devices, micro-mixers are in common use in biological and chemical analyses for $\mu$-TAS practice. Numerous studies have discussed improvement of the efficiency for various micro-mixers. Nearly all these studies exploited syringe pumps as driving sources to transport the test fluids [1-7]. Those pumps require either complicated fabrication processes or external energy sources like electrical power, which can limit the operation of fluidic devices. The use of rechargeable batteries may resolve the issue; however, this causes additional environmental concerns [8-12]. Several researchers have also devoted substantial effort to designing the micro-mixers with the features of three-dimensional chambers

* Corresponding author. Address: Department of Mechanical Engineering National Taiwan University, No. 1, Sec. 4, Roosevelt Road, Taipei 10617, Taiwan, ROC. Tel.: +886 23366 2689; fax: +886 223631755 .

E-mail address: phchen@ntu.edu.tw (P.-H. Chen). for mixing enhancement [13-17]. Nevertheless, these designs have crucial shortcomings such as complex chamber configuration with multiple inlets and outlets, high fabrication cost due to the use of Micro-Electro-Mechanical Systems (MEMS) technology, and long reaction time attributable to the flow process at low Reynolds numbers (Re).

Most early micro-mixers lack a self-driving feature and require electric power during utilization. This study uses finger-driven electricity free pumps, as Liou et al. [18] presented, to mitigate the risk of single-point failure in delivering test fluids to mixing devices. Accordingly, polydimethylsiloxane (PDMS) made components can be fabricated and integrated into different modular devices (including tube, pump, mixer, valve and reservoir), which allow ready construction of a fully-portable, disposable fluidic system. Additionally, to realize rapid mixing and bio-reaction of two liquids under high-Re operational conditions, this study develops a vortex mixer with a plain hemisphere-shaped structure using the simplest design of two inlets and one outlet, and the mold procedure rather than the elaborate MEMS process, to replicate any desired mixer-chamber shape. As an effective tool to characterize 


\begin{tabular}{|llll|}
\hline Nomenclature & & \\
$C$ & molar concentration of the species & $t$ & time \\
$C_{i}$ & species concentration profile & $u_{i}$ & velocity component in the $i$ direction \\
$\bar{C}$ & an average of the species concentration profile & $u_{j}$ & velocity component in the $j$ direction \\
$D$ & binary diffusion coefficient of the species & $V$ & fluid velocity \\
$D_{h}$ & hydraulic diameter of the channel & $W$ & outlet diameters of the mixer \\
$H$ & maximum axial displacement & $x_{i}$ & coordinates component in the $i$ direction \\
$L$ & distance from mixer chamber center to outlet & $x_{j}$ & coordinates component in the $j$ direction \\
$N$ & number of pixels in the image & $\mu$ & fluid viscosity \\
$p$ & pressure & $\rho$ & fluid density \\
$\operatorname{Re}$ & Reynolds number & $\eta$ & mixing index \\
$T$ & time period for one push by a cam & & \\
& & & \\
\hline
\end{tabular}

the inherently convoluted transport phenomenon inside the mixer, a commercial computational fluid dynamics (CFD) software CFDACE $+{ }^{\circledR}$ conducted numerical computations (CFD-ACE $+{ }^{\circledR}$ 2010).
Both measurements and simulations examined mingling-progression performance of the proposed mixer in a one-shot mixing event. Numerical analysis studied the differences in pressing

(a)

\section{Placing}

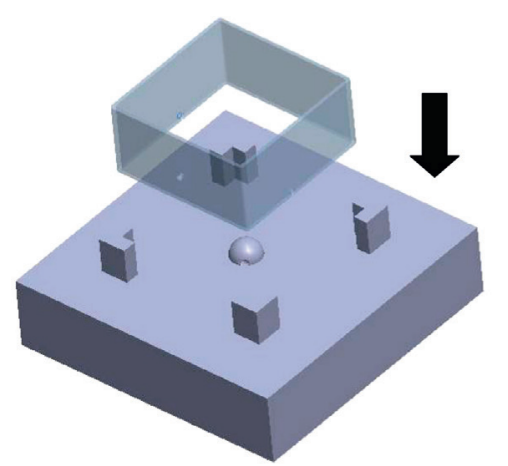

Pouring PDMS

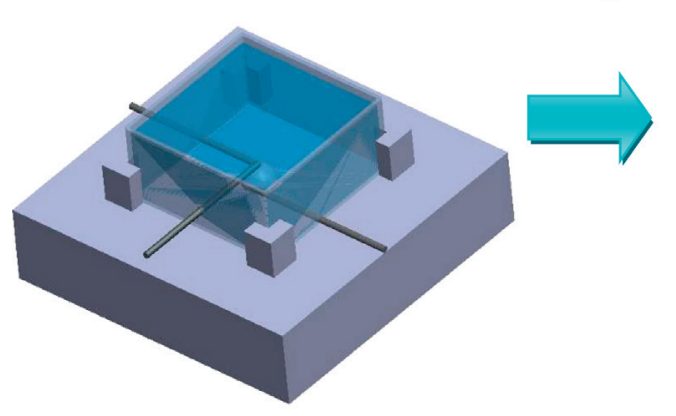

Inserting metal rods

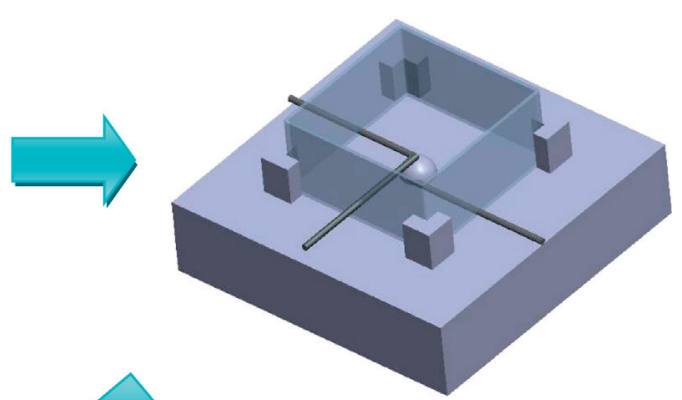

Assembling

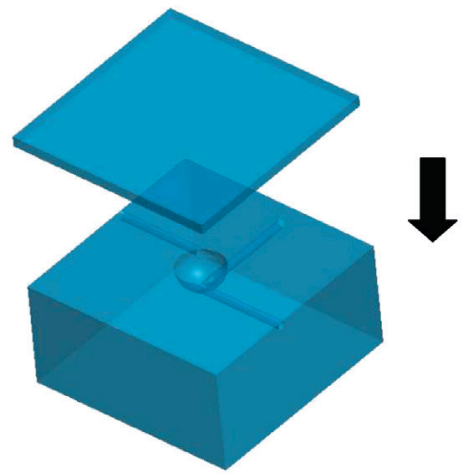

(b)

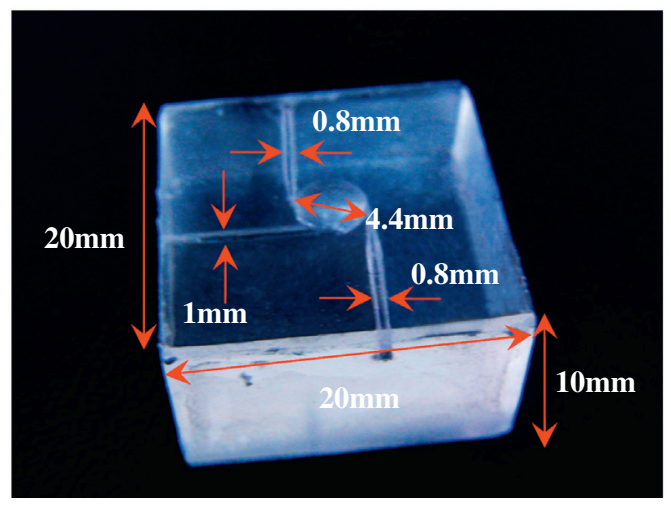

Fig. 1. (a) Schematic of the fabrication process and (b) picture of a vortex mixer. 
displacement (with the same time interval at a push displacement of less 20\%) and time delay (based on the equal displacement with a $20 \%$ increase in time period) of two human-powered pumps on mixer efficiency.

\section{Design and fabrication of pump and vortex mixer}

All components in this study were made of PDMS with the related design and fabrication methods described below. Fig. 1 shows (a) a schematic of the fabrication process and (b) a picture of the vortex mixer. The mixer consisted of three sections: a hemispherical chamber, two inlets and one outlet allowing the liquid passage, and the inlets and outlet were located on two sides and the bottom of the chamber. The dimensions of the mixer were $20 \times 20 \times 10 \mathrm{~mm}$ with a $4.4-\mathrm{mm}$ chamber diameter to confirm biological applications; whereas the diameters and lengths of the inflow and outflow channels were $0.8 \times 10 \mathrm{~mm}$ and $1.0 \times 10 \mathrm{~mm}$, respectively. A two-piece assembled casting design permitted researchers to easily strip the outer mold down while retaining the product. A mixture of PDMS prepolymer and curing agent $\left(10: 1 \mathrm{w} / \mathrm{w}\right.$, Sylgard ${ }^{\circledR} 184$ silicone elastomer, Dow Corning) was first prepared and poured into the aluminum mold with the container cured at $100^{\circ} \mathrm{C}$ for $45 \mathrm{~min}$ to construct the mixing chamber.

Adopting the design from Liou et al. [18], PDMS elasticity permits development of a suction pump. The original truncatedcone-shaped pump chamber could cause a dead volume at the periphery when the chamber is fully compressed. Hence, the current pump was developed with the improved features of a hemisphere-shaped chamber to minimize dead space (with the dead-volume ratio down to $6 \%$ ) and a thicker top diaphragm of the pump to enhance durability. The pumps were fabricated using a casting method similar to the mixer's process. Fig. 2 displays (a) a picture and (b) a schematic of the finger-pressed pump. The hemispherical pump chamber diameter was $11 \mathrm{~mm}$ for ergonomic

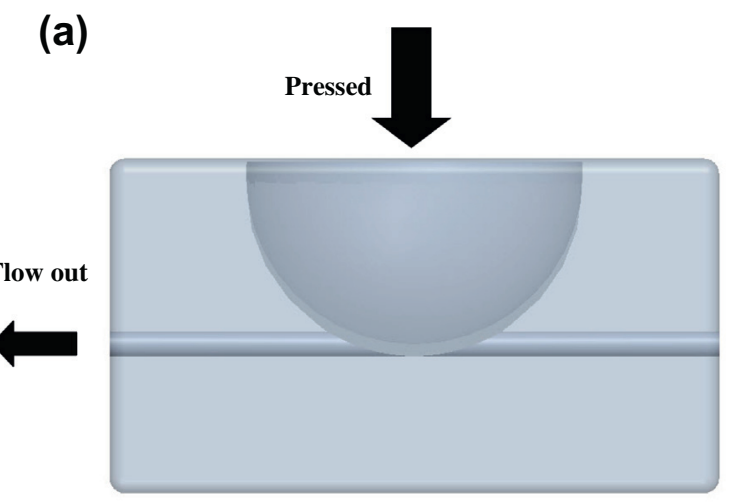

(b)

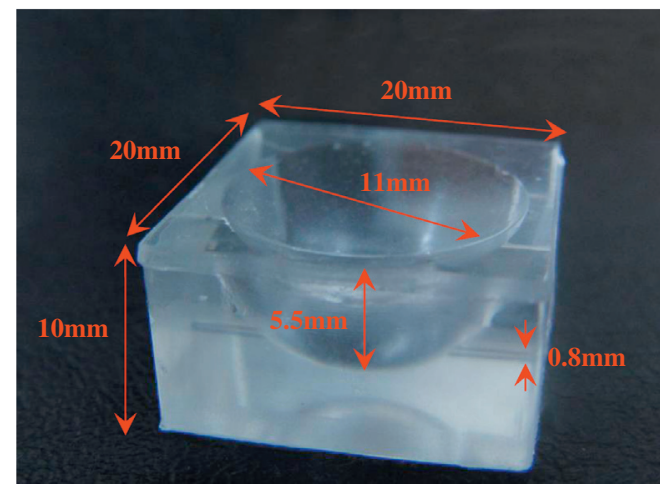

Fig. 2. (a) Schematic and (b) picture of a finger-pressed pump. reasons, whereas inlet and outlet diameters were both $0.8 \mathrm{~mm}$. In practice, polyethylene tubes (INTRAMEDIC) with inner and outer diameters of 0.580 and $0.965 \mathrm{~mm}$, respectively, connected the pumps and the mixer. Elastic PDMS was used to clamp the tube tightly, with no observed leakage during the experiments.

\section{Experimental apparatus}

Fig. 3 illustrates a schematic of the experimental setup established to investigate the flow-field and mixing performance of the proposed vortex mixer. Two cams driven by a rod-motor system, rather than human fingers, generated hydrodynamic forces delivering nearly the same liquid flow-rates from two pumps as the baseline study case. The cam profile was constructed according to the kinematic inversion principle to emulate the average volumetric flow-rate discharged from a human finger-pressed pump.

This study used two liquid samples for mixing: de-ionized (DI) water and a DI water solution containing green dye $(0.1 \% \mathrm{w} / \mathrm{w})$ (fluorescein sodium salts diluted in water, SIGMA-ALDRICH). The experiments used a fluorescence microscope (BH2-UMA, OLYMPLUS, JAPAN) with an objective lens ( $M$ plan APO series $M=100$, $\mathrm{NA}=0.42$ ). The light from a mercury-arc lamp was directed through a set of epi-optical filters $(532 \mathrm{~nm})$. A CCD color telescope camera $(1024 \times 768$ pixels, 8-bit color DFK 31AU03.AS, IMAGINGSOURCE, Germany) acquired optical images, which were then processed with an image-acquisition and data-processing board (DOMINO Harmony), displayed on a monitor, then stored as a file on a personal computer. Those captured images are transformed to the gray-scale images for calculating mixing efficiency. The image gray level of the fluid can represent the fluid concentration. If the relation between the gray level and the concentration is linear, we can then use the numerical value of the gray level for concentration evaluation. Supposing the gray levels of the pure fluid $A$ and $B$ are $C_{A}$ and $C_{B}$, respectively, the concentration of the complete mixture is $\bar{C}=\alpha C_{A}+(1-\alpha) C_{B}$ for the case that the volume fraction of the fluid $A$ in the mixture is $\alpha$. Consider the following absolute deviation of the gray levels of the $N$ pixels in the graphic image:

$\zeta \equiv \frac{1}{N} \sum_{i=1}^{N}\left|C_{i}-\bar{C}\right|$

The symbol $C_{i}$ denotes the species concentration profile across the transverse direction at varying axial distances from the entrance. When two fluids are completely mixed, the concentration is homogeneous and $C_{i}=\bar{C}$ for all pixels. The corresponding $\zeta$ is equal to zero. On the other hand, for this non-mixing state in the very beginning of the mixing process, the images should contain $\alpha N$ pixels with $C_{A}$ and $(1-\alpha) N$ pixels with $C_{B}$. The corresponding $\zeta$ has the maximum value $\left(\zeta_{M}\right)$ expressed as below.

$\zeta_{M}=2 \alpha(1-\alpha)\left|C_{A}-C_{B}\right|$.

Now we can define an index for mixing efficiency $\eta$ as follows:

$\eta \equiv 1-\frac{\zeta}{\zeta_{M}} \equiv 1-\frac{1}{2 \alpha(1-\alpha) N} \sum_{i=1}^{N} \frac{\left|C_{i}-\bar{C}\right|}{\left|C_{A}-C_{B}\right|}$.

For uniform mixing, concentration deviation should be 0 with a mixing index of 1 . Conversely, decreasing the mixing index from 1 to 0 achieves a lesser mixing amount. A " 0 " mixing index stands for the species concentrations in a completely unmixed state.

\section{Numerical analysis}

A theoretical model implemented in the CFD-ACE $+{ }^{\circledR}$ software simulates the mixing process of two different test fluids in the 
proposed vortex mixer. To maintain consistent experimental conditions, one test liquid was a solution of green dye in DI water, and the other was DI water. In the analysis, both fluids were treated as incompressible, laminar, miscible liquid flows with uniform properties, and considering the insignificant effects of gravity, chemical reaction and temperature variation over the computational domain. The model was based on the time-dependent three-dimensional conservation equations of mass, momentum, and species concentration with the mathematical expressions that follow.

$\frac{\partial u_{i}}{\partial x_{i}}=0$

$\frac{\partial}{\partial t}\left(\rho u_{i}\right)+\frac{\partial\left(\rho u_{i} u_{j}\right)}{\partial x_{j}}=-\frac{\partial p}{\partial x_{i}}+\frac{\partial}{\partial x_{j}}\left[\mu\left(\frac{\partial u_{i}}{\partial x_{j}}+\frac{\partial u_{j}}{\partial x_{i}}\right)\right]$,

$\frac{\partial}{\partial t} C+\frac{\partial}{\partial x_{j}}\left(u_{j} C\right)=\frac{\partial}{\partial x_{j}}\left(D \frac{\partial C}{\partial x_{j}}\right)$.

The symbol $u_{i}$ denotes the velocity component in the $i$ direction; moreover, $p, \rho, \mu, D$ and $C$ represent the pressure, density, dynamic viscosity, binary diffusion coefficient and molar concentration of the species. The physical properties of pure water were applied to two test fluids, and the corresponding values of $\rho, \mu$ and $D$ were $1000 \mathrm{~kg} / \mathrm{m}^{3}, 0.001 \mathrm{~N}-\mathrm{s} / \mathrm{m}^{2}$, and $10^{-10} \mathrm{~m}^{2} / \mathrm{s}$, respectively [19].

The normalized concentration of one of the fluid species was set to 0 and the other to 1 at the inlets. In calculations, $\operatorname{Re}=\rho V D_{h} / \mu$ and $D_{h}$ is the diameter of the inflow channels. At the outlet, a fixed pressure of $1 \mathrm{~atm}$ was prescribed and the flow developed fully with all primitive flow variables extrapolated from the interior values. The no-slip condition and the zero normal gradients of pressure and species concentration were imposed on the solid walls of the vortex mixer. To arrange the initial condition for investigating the transient mixing process, the mixer was filled with still DI water (species concentration of 0 ) in simulations. In the channel entry, one of the feed streams introduced DI water with green dye (species concentration of 1 ) at the beginning of one push of the pumps. When the streams flowed into the mixer chamber, the mingling progression of two miscible liquids developed a rolling mixing surface in the center region with complete mixing of two liquids realized at the normalized concentration of 0.5 . To assess mixer performance, the same equation for the experiments calculated mixing efficiency (Eq. (3)).

These mathematical equations were discretized into a set of cells through the finite control volume approach with an iterative semi-implicit method for pressure-linked equations consistent

\section{Acquire and Control System}

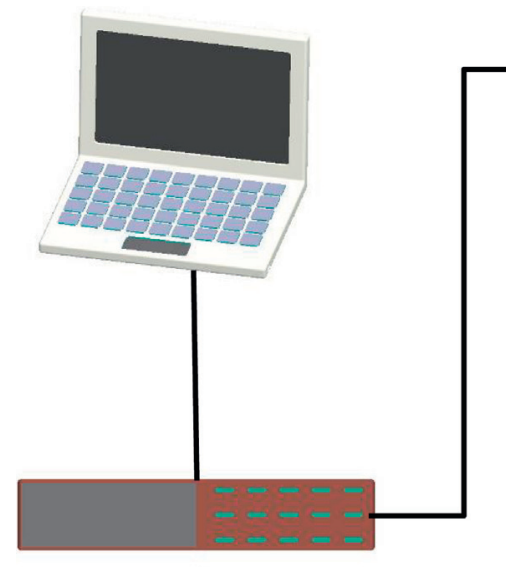

Synchro-timing Hub

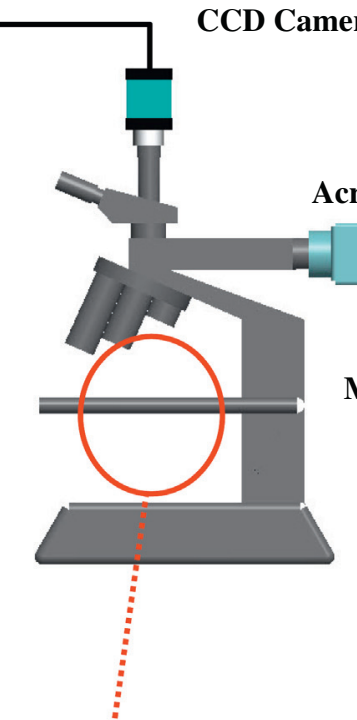

Acr-lamp with filter

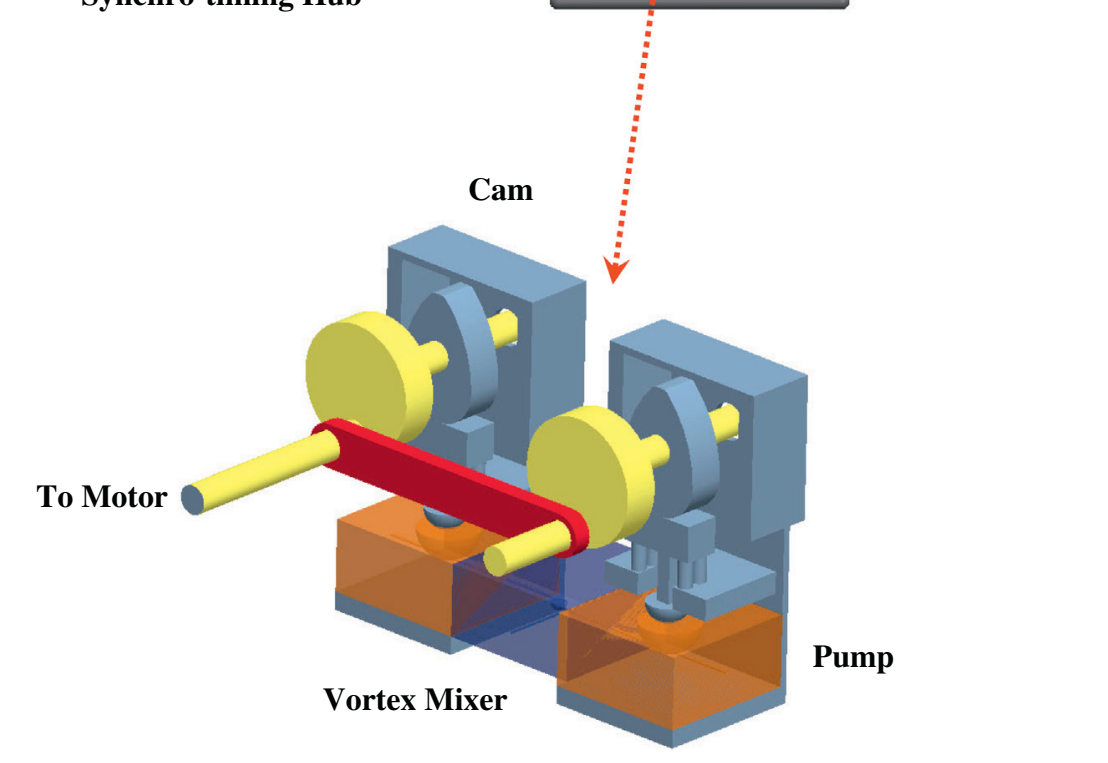

Fig. 3. Schematic of the experimental setup for measurements of mixing performance. 
(SIMPLEC) numerical scheme implemented to couple the velocity and pressure [20]. The dispersion-time evolution of one test fluid into another was calculated explicitly with a stability requirement on the allowable time-step magnitude. In each numerical cell, the stability limit was restricted by the Courant-Friedrichs-Lewy (CFL) number, defined as (the local velocity magnitude) $\times$ (the maximum time-step)/(the length of a local cell), which must satisfy the condition of a CFL number lower than one by setting the automatic time-step option in the CFD-ACE $+{ }^{\circledR}$ software.

\section{Results and discussion}

To examine the mixing phenomenon for two test fluids flowing into a common chamber via two inlets, Fig. 4 shows (a) a photograph and (b) numerical grids of the vortex mixer for mixing simulations. The mixer mesh setup displayed unsteady calculations conducted using the $\mathrm{ACE}+{ }^{\circledR}$ software on total grids of 188,610 , 254,624 , and 341,196 points at the fixed time steps of 0.001 and $0.0005 \mathrm{~s}$. The mesh setup consists of six structured sections, including the mixing chamber, two pumps, two inflow channels and one outflow channel. Finer grids were placed in the core, joint and near wall regions of the chamber and pumps, having the smallest spacing of about $14.9 \mu \mathrm{m}$ for resolving steep flow-property variations. During the calculations for each time step, the normalized residual errors of flow variables converged to $10^{-5}$ with the mass conservation check within $0.5 \%$. The calculated results of the transitory profiles of velocity magnitude and species concentration along the centerline of the chamber at different grids and time steps suggested that satisfactory grid independence is possible through a mesh setup of 254,624 grids with a time step of $0.001 \mathrm{~s}$. A full simulation for advancing a one-shot mixing event in a 0.65 -s period generally requires $250 \mathrm{~h}$ of central processing

\section{(a)}

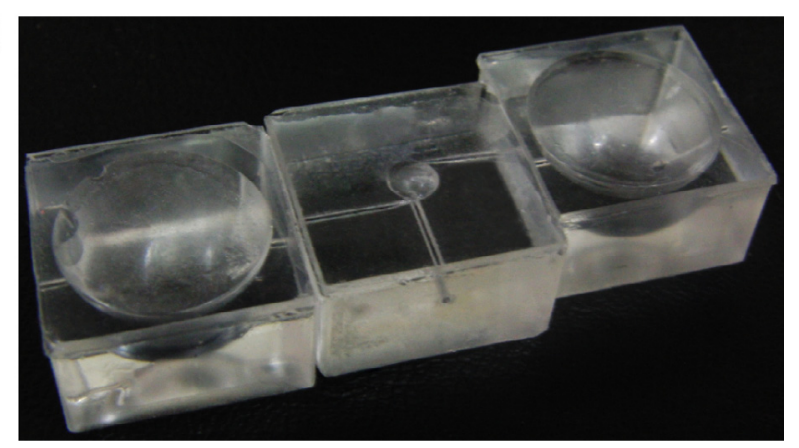

(b)

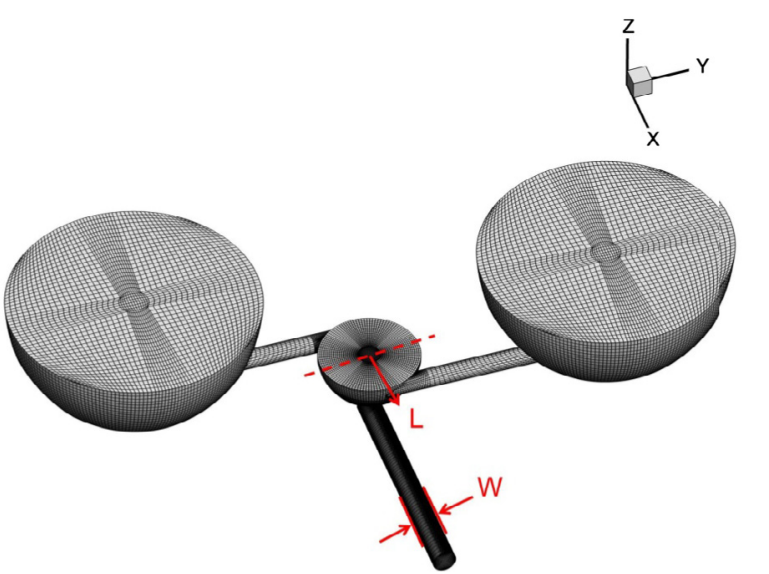

Fig. 4. (a) Photograph and (b) numerical grids of the vortex mixer for mixing simulations.

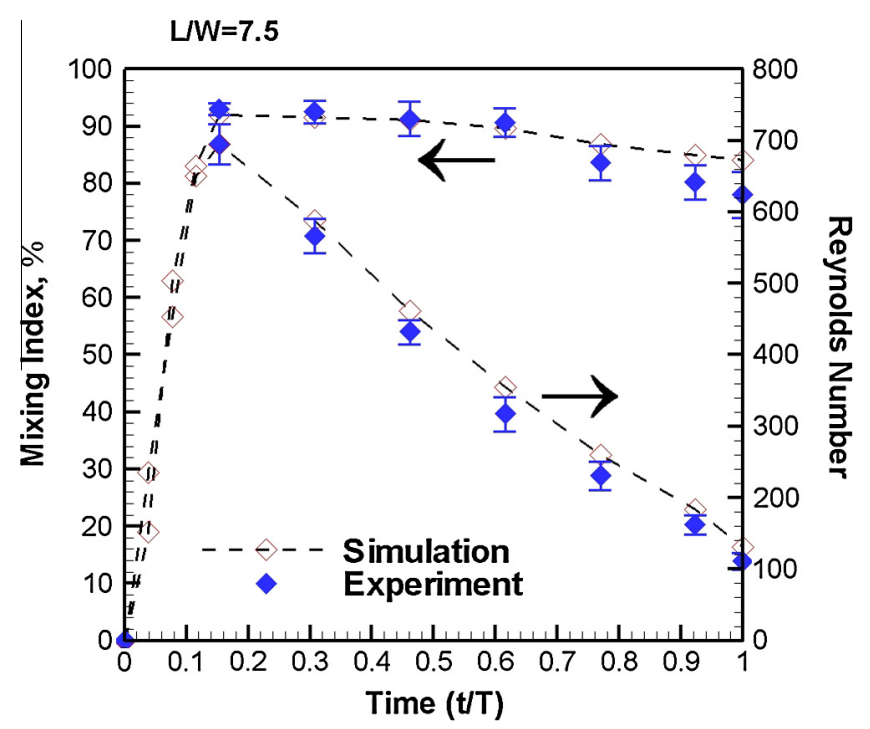

Fig. 5. Comparison of the predicted time histories of mixing index and Reynolds number with experimental data. (a)

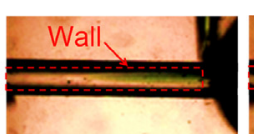

$t / T=0.038$

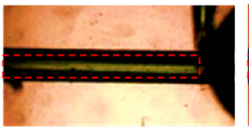

0.115

(b)
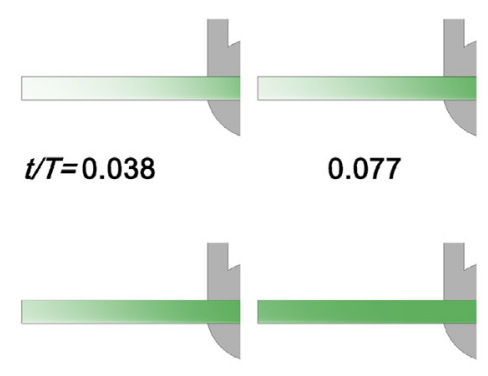

0.115

0.154

Molar Concentration (c)
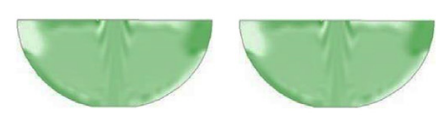

$t / T=0.154$

0.308
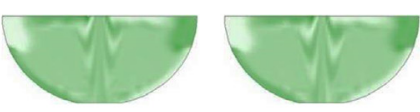

0.769

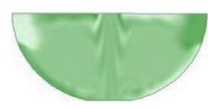

0.462

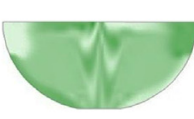

0.923

Fig. 6. Comparison of (a) visualized images with predicted time-varying concentration distributions in (b) top and (c) side views describing the mixing development. 
unit time on an Intel ${ }^{\varpi 0} \operatorname{Core}^{\mathrm{TM}} 2$ Quad Q9550-2.83 GHz personal computer.

Considering the vortex mixer run by two simultaneous fingerpressed pumps without electrical power, Fig. 5 compares the predicted non-dimensional time histories of mixing index and Re with the experimental data for a one-shot mixing event. $L$ is the distance from mixer chamber center to outlet; $W$ is outlet diameters of the mixer $(1 \mathrm{~mm})$. Measuring the volume variation of water from the pump outlet makes it possible to determine the system Re Lee et al. [21]. Each data point represents the averaged value of over five measured records with the error bars designated by $\pm 3 \sigma_{s d}$, where $\sigma_{s d}$ is the standard deviation. Initially filling the vortex mixer, the test liquids were promptly pushed out of the pumps. Responding to the cam pressing the PDMS diaphragm downward for each pump at $t=0$, they streamed into the mixing chamber through the inflow channels. For the specified flow condition controlled by the cam profile during the tests, the fluids quickly reached the maximum discharged flow-rate with the instant predicted and measured peak Re of 695 and 677 attained at $t /$ $T=0.154(t=0.1 \mathrm{~s} ; T=0.65 \mathrm{~s})$. In this early stage, high-Re inflows intensely augmented the strength of swirling vortices in the mixer chamber, and thereby reached a good mixing index $(\eta)$ of $93 \%$ at $t /$ $T=0.154$. Thereafter, expelled flow-rates continually decreased to the end of one-shot push with the ensuing Re below 140 at the end of pressing duration. Meanwhile, the vortex blending performance progressively deteriorated in response to the decrease of Re with $\eta=0.8$ at $t / T=1.0$. However, both experimental and simulation results show that better mixing could be retained in the period from $t / T=0.154$ to 0.615 (with $\eta=0.9$ at $t / T=0.615$ ) during the transient mixing progression. In general, the predictions agree reasonably well with the experimental results of the mixing index and Re with the errors of $1.1 \%$ and $3.4 \%$, respectively, at $t / T=0.462$, indicating that the simulation software can predict the mixing flowfield with an adequately accuracy.

To depict the species concentration field, Fig. 6 compares (a) visualized images with predicted time-varying concentration distributions in (b) top and (c) side views describing the mixing progression at different times. The dim images in the outlet of the mixer revealed the blending progression of the green dye and water from $t / T=0.038$ to 0.154 ; whereas the green dye and water nearly completely mixed after $t / T=0.154$. Constrained by the inner wall of the hemispherical chamber, Fig. $6 \mathrm{c}$ shows how the two streams tended to blend together along the twirl course toward a confluent center over the top, and wound downwards to form a solid swirling vortex. The fading color difference indicated that the swirling flow induced significant mixing occurrence from the top area to the bottom area with a full color of light green appearing on the outlet section. The corresponding concentration of $0.5 \mathrm{dem}-$ onstrates a well-mixed state during the period of $t / T=0.154-0.615$ in this case. Accordingly, the decline of Re produced the dilution of rotating vortex strength, and thereby led to a relatively non-uniform species concentration distribution. In general, the predicted non-dimensional time sequences of concentration contours agree reasonably well with the observed images.

Fig. 7 illustrates the predicted time-varying velocity vectors, magnitude contours and streamlines in (a) top and (b) side views during the mixing period of $t / T=0.154-0.923$. As inflow velocities peaked at $t / T=0.154$, the liquids swirled strongly around the center in a coil-like fashion, distorting and stretching fluid interfaces to produce chaotic advection. as a result, the enlarged interfacial area, across which diffusion occurs between the fluids, can enhance the mixing effect. However, a relatively weaker twisting vortex can be observed in the core due to the declining supply flow-rates when $t / T \geqslant 0.154$

Fig. 8 presents the predicted time-varying streamlines and concentration distributions in stereogram view during the early (a)
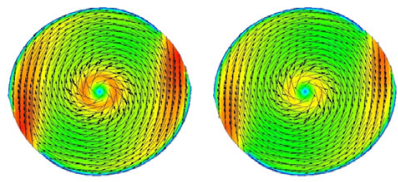

$t / T=0.154$

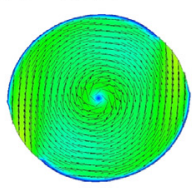

0.615

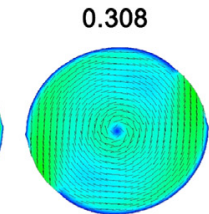

0.769

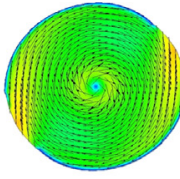

0.462

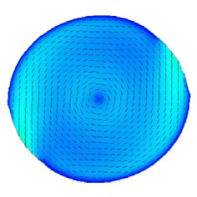

0.923

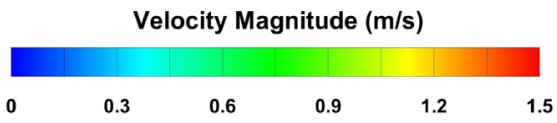

(b)
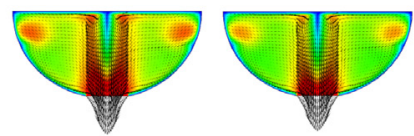

$t / T=0.154$
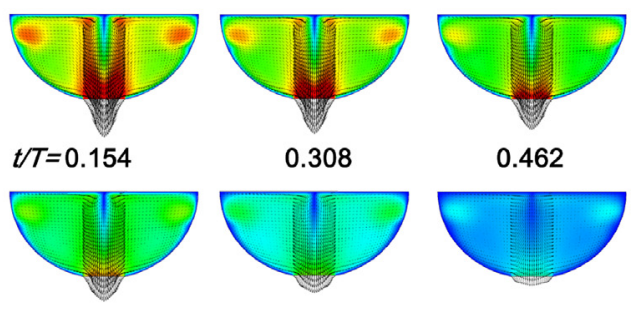

0.615

0.769

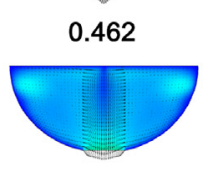

0.923

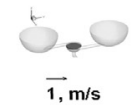

$\overrightarrow{1, m / s}$
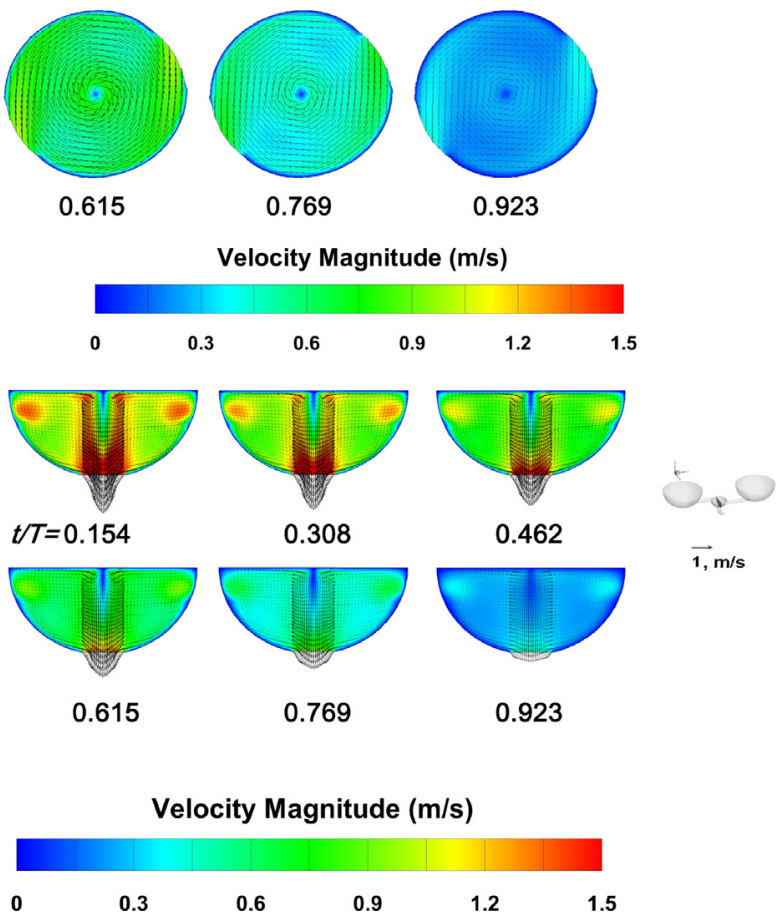

Fig. 7. Predicted time-varying velocity vectors, magnitude contours and streamlines in (a) top and (b) side views during the mixing period of 0.1-0.6 s.

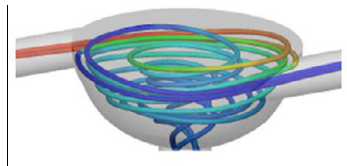

$t / T=0.031$

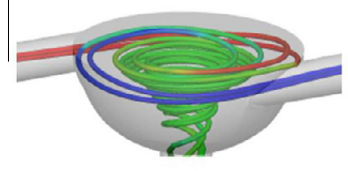

0.154

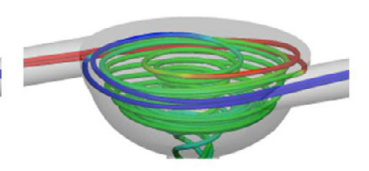

0.092

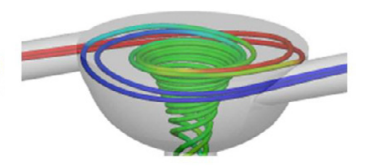

0.231

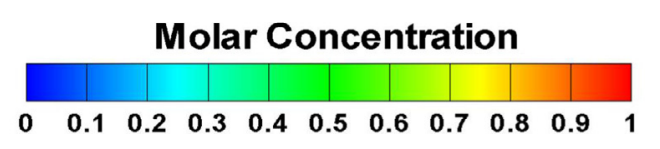

Fig. 8. Predicted time-varying streamlines and concentration distributions in stereogram view during the early mixing stage of $0.02-0.15 \mathrm{~s}$.

mixing stage of $t / T=0.03-0.23$. The trace lines started at two entrances and followed the flow course to the mixer exit with the line colors signifying the species concentration values. At the very start of a pumping event, two test liquids were run into the vortex mixer with the corresponding colors of red and blue (i.e., concentrations of 1 and 0 ) near the channel entries. Two fluid 


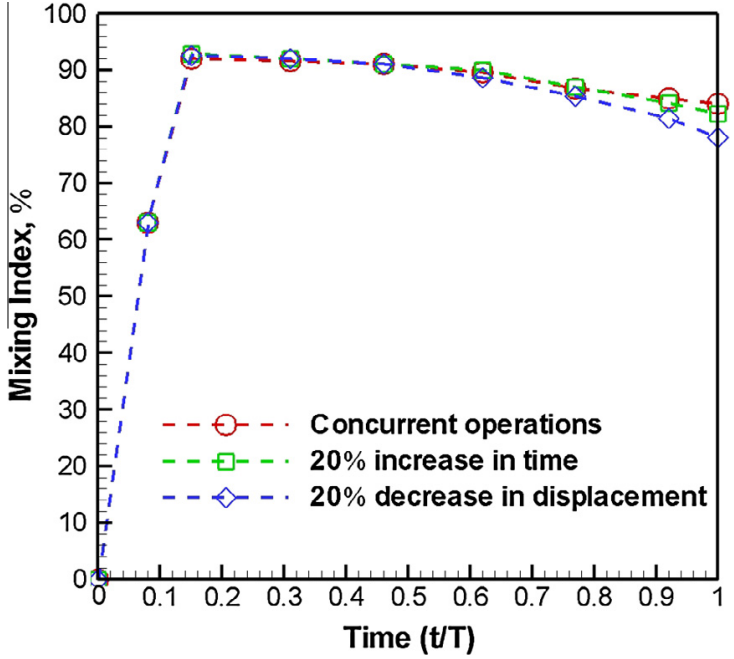

Fig. 9. Effects of the differences in pressing displacement and time duration of two human-powered pumps on the mixing index.

streams then rolled downward and produced a weak vortex structure due to a hemispherical shape of the mixer. Because the mixer was first loaded with water, the unmixed fluid was quickly discharged with the species concentration value of approximately 0.1 on average near the outlet at $t / T=0.03$. After the green-dyed water circulated throughout the mixer at $t / T=0.09$, a developed fast revolving motion was expected to sustain the rolling vortex structure for achieving effective mixing. In effect, the rotating streamlines clearly revealed an induced three-dimensional twisting vortex in the mixer. At $t / T \geqq 0.154$, the concentration of 0.5 (in green color) at the exit indicated complete mixing of the test fluids.

In practice, the variations of working parameters can alter the fluid mixing performance of a vortex mixer. Fig. 9 shows the effects of different pressing displacements and time durations for two human-powered pumps on the mixing index. During operations, two finger-pressed pumps of the proposed mixer may not concurrently function at exactly same statuses with one push. Providing the 5.5$\mathrm{mm}$ axial displacement and $0.65 \mathrm{~s}$ time duration for the state of the first pump, the second pump operated in two cases: one for the same time interval at a push displacement of less $20 \%$, and another for equal displacement with a $20 \%$ increase in time period. Differences were visible when $t / T \geqq 0.769$. However, the simulated results indicate that the proposed mixer with the variations up to $20 \%$ in displacement and duration may still produce a satisfactory mixing performance within $t / T=0.615$.

\section{Conclusions}

This study presents the design, fabrication and operation of a geometrically simple vortex mixer actuated by one-shot electricity-free pumps. It examines the rapid mixing process occurring in the mixer via numerical calculations and experimental measurements. During the transient mixing process, two test liquids tangentially flowed into the mixer along the inner wall of the hemisphere chamber. The fluids rapidly achieved the greatest delivered flow rate with the measured peak Re of 677 at $t /$ $T=0.154(t=0.1 \mathrm{~s})$. Those two streams tended to blend together along the coiled passage toward the center, and wound downwards to generate a solid swirling vortex. The vortex agitation could further fold and stretch the fluids spirally to increase both interfacial area and mixing efficiency. Since the high-Re inflows strongly augment the vortex strength for mixing enhancement, the current vortex mixer merely needs $0.1 \mathrm{~s}$ to attain $\eta$ of $93 \%$ and maintains a good mixing performance during the period of 0.1-0.4 s for a one-shot event. This investigation also studies the influence on mixing effectiveness of differing displacement and duration of two human-powered pumps for one manual push. Variations of displacement and duration (up to $20 \%$ ) do not deteriorate the mixing effect within $0.4 \mathrm{~s}$ for the mixer.

\section{Acknowledgments}

The authors would like to thank the National Science Council of Taiwan, Republic of China, for their financial support under Contract Numbers NSC 99-2221-E-002-119-MY3 and NSC 99-2221E-002-081-MY3 and NSC 101-2221-E-027-148.

\section{References}

[1] D. Ahmed, X. Mao, J. Shi, B.K. Juluria, T.J. Huang, A millisecond micro-mixer via single-bubble-based acoustic streaming, Lab Chip 9 (2009) 2738-2741.

[2] M.A. Ansari, K.Y. Kim, K. Anwar, S.M. Kim, A novel passive micro-mixer based on unbalanced splits and collisions of fluid streams, J. Micromech. Microeng. 20 (2010) 055007-055016.

[3] A. Soleymani, E. Kolehmainen, I. Turunen, Numerical and experimental investigations of liquid mixing in T-type micromixers, Chem. Eng. J. 135 (2008) 219-228.

[4] M. Kashid, A. Renken, L. Kiwi-Minsker, Mixing efficiency and energy consumption for five generic microchannel designs, Chem. Eng. J. 167 (2011) 436-443.

[5] L. Li, L.J. Lee, J.M. Castro, A.Y. Yi, Improving mixing efficiency of a polymer micro-mixer by use of a plastic shim divider, J. Micromech. Microeng. 20 (2010) 035012-035020.

[6] Y. Fang, Y. Ye, R. Shen, P. Zhu, R. Guo, Y. Hu, L. Wu, Mixing enhancement by simple periodic geometric features in microchannels, Chem. Eng. J. 187 (2012) 306-310.

[7] T.H. Tsai, D.S. Liou, L.S. Kuo, P.H. Chen, Rapid mixing between ferro-nanofluid and water in a semi-active Y-type micro-mixer, Sens. Actuat. A 153 (2009) 267-273.

[8] H. Tajima, Y. Yoshida, S. Abiko, K. Yamagiwa, Size adjustment of spherical temperature-sensitive hydrogel beads by liquid-liquid dispersion using a Kenics static mixer, Chem. Eng. J. 156 (2010) 479-486.

[9] C. Galletti, M. Roudgar, E. Brunazzi, R. Mauri, Effect of inlet conditions on the engulfment pattern in a T-shaped micro-mixer, Chem. Eng. J. 185-186 (2012) 300-313.

[10] E. Saatdjian, A.J.S. Rodrigo, J.P.B. Mota, On chaotic advection in a static mixer Chem. Eng. J. 187 (2012) 289-298.

[11] M. Hammoudi, E.K. SI-Ahmed, J. Legrand, Dispersed two-phase flow analysis by pulsed ultrasonic velocimetry in SMX static mixer, Chem. Eng. J. 191 (2012) 463-474.

[12] J.M. Park, K.D. Seo, T.H. Kwon, A chaotic micro-mixer using obstruction-pairs, J. Micromech. Microeng. 20 (2009) 015023-015033.

[13] Y.C. Chung, Y.L. Hsu, C.P. Jen, M.C. Lu, Y.C. Lin, Design of passive mixers utilizing microfluidic self-circulation in the mixing chamber, Lab Chip 4 (2004) 70-77.

[14] C.H. Lin, C.H. Tsai, L.M. Fu, A rapid three-dimensional vortex micro-mixe utilizing self-rotation effects under low Reynolds number conditions, J. Micromech. Microeng. 15 (2005) 935-943.

[15] M. Long, M.A. Sprague, A.A. Grimes, B.D. Rich, M. Khine, A simple threedimensional vortex micro-mixer, Appl. Phys. Lett. 94 (2009) (133501-1-3).

[16] S.Y. Yang, J.L. Lin, G.B. Lee, A vortex-type micro-mixer utilizing pneumatically driven membranes, J. Micromech. Microeng. 19 (2009) 035020-035028.

[17] M.A. Ansari, K.Y. Kim, K. Anwar, S.M. Kim, Vortex micro T-mixer with nonaligned inputs, Chem. Eng. J. 181-182 (2012) 846-850.

[18] D.S. Liou, Y.F. Hsieh, L.S. Kuo, C.T. Yang, P.H. Chen, Modular component design for portable microfluidic devices, Microfluid Nanofluid 10 (2011) 465-474.

[19] A.A.S. Bhagat, E.T.K. Peterson, I. Papautsky, A passive planar micro-mixer with obstructions for mixing at low Reynolds numbers, J. Micromech. Microeng. 17 (2007) 1017-1024.

[20] J.P. Van Doormaal, G.D. Raithby, Enhancements of the SIMPLE method for predicting incompressible fluid flows, Numer. Heat Trans. 7 (1984) 147-163.

[21] C.H. Chiou, G.B. Lee, Minimal dead-volume connectors for microfluidics using PDMS casting techniques, J. Micromech. Microeng. 14 (2004) 1484-1490. 